\title{
Case - Azoospermia with bilateral varicocele and normal follicle-stimulating hormone
}

John Michael Masterson; Ranjith Ramasamy

Department of Urology, University of Miami Miller School of Medicine, Miami, FL, United States

Cite as: Can Urol Assoc J 2018 June 8; Epub ahead of print. http://dx.doi.org/10.5489/cuaj.5298

\section{Published online June 8, 2018}

***

\section{Introduction}

Azoospermia affects approximately 15\% of infertile men and can be caused by obstructive azoospermia (OA), non-obstructive azoospermia (NOA). ${ }^{1}$ Azoospermia can also be caused by ejaculatory dysfunction such as retrograde ejaculation or anejaculation, which fall outside the realm of OA or NOA.OA accounts for approximately $40 \%$ of cases of azoospermia and is caused by anatomical obstruction of the extra-testicular ductal system anywhere between the rete testis and the ejaculatory ducts. ${ }^{2}$ Physical blockages resulting in $\mathrm{OA}$ are most commonly due to prior vasectomy, but may also be caused by epididymal, vasal, or ejaculatory duct pathology secondary to genitourinary infection, iatrogenic injury during scrotal or inguinal surgical procedures, and congenital anomalies such as congenital bilateral absence of the vas deferens (CBAVD). ${ }^{3}$ NOA on the other hand accounts for $60 \%$ of cases of azoospermia and is caused by either primary or secondary testicular failure and subsequent defective spermatogenesis. ${ }^{4}$ Varicocele is considered a correctable cause of NOA. ${ }^{5}$

Management of OA typically involves surgical treatment with the goal of returning sperm to the ejaculate. Microsurgical reconstruction techniques for OA include vasovasostomy (VV), vasoepididymostomy (VE), and transurethral resection of the ejaculatory duct (TURED). ${ }^{6} \mathrm{VV}$ is $57 \%-96 \%$ successful depending on the type of procedure while VE is $78 \%$ successful. ${ }^{6}$ If reconstruction is not possible as in the case of CBAVD, microsurgical aspiration of epididymal sperm for assisted reproductive techniques (ART) is an option. Approximately $10 \%$ of men with NOA will have sperm in the ejaculate upon subsequent testing, however for the remaining $90 \%$ management of NOA almost always involves ART in the form of microdissection testicular sperm extraction (mTESE) in combination with in vitro fertilization/ intracytoplasmic sperm injection (IVF/ICSI), as return of sperm to the ejaculate is less likely overall in NOA. ${ }^{4} \mathrm{mTESE}$ carries an overall success rate of $52 \%$. ${ }^{7}$ For men with NOA and varicocele, varicocele repair is a possible solution, with reported success rates of $39 \%$ for return of sperm to the ejaculate and 11.3\%-54.5\% for mTESE sperm retrieval depending on underlying histology. ${ }^{8}$ Given the considerable differences in etiology and management strategies between OA and NOA, accurate diagnosis of these conditions is important. 
Per current American Urological Association (AUA) and Canadian Urological Association (CUA) guidelines, the initial evaluation of an azoospermic patient should include a full medical history, physical examination, and measurement of serum testosterone and folliclestimulating hormone (FSH) levels. ${ }^{5,9}$ Relevant historical factors in the evaluation of azoospermia are listed in Table $1 .{ }^{5}$ Positive physical exam findings for men with OA include large testis (the normal adult testicle should be approximately 15-20cc), enlarged or indurated epididymis, epididymal cysts, abnormal digital rectal exam (DRE), or absence of the vas deferens. ${ }^{10}$ Positive physical exam findings suggestive of NOA include small testis (less than $15 \mathrm{ml}$ ), flat epididymis, or varicocele without evidence of obstruction. ${ }^{11}$ Unfortunately there can be overlap in physical exam findings among men with azoospermia.

Measurement of FSH and testis volume is extremely useful in the evaluation of azoospermia. In 2002, Schoor et al. found that $96 \%$ of cases of OA and $89 \%$ of cases of NOA could be diagnosed based on these parameters alone: with OA having normal FSH and testis volume; and NOA having elevated FSH and low testis volume. ${ }^{12}$ These findings are reflected in current guidelines on the evaluation of azoospermia and have greatly reduced the need for testicular biopsy in the evaluation of azoospermia. ${ }^{5,9}$ The purpose of this case report is to discuss a diagnostically challenging presentation of azoospermia in the context of current recommendations and to propose a situation-specific treatment algorithm to aid in the management of azoospermia and varicocele.

\section{Case report}

We present the case of a 43-year-old male who presented to a tertiary academic urology clinic for evaluation of primary infertility. Semen analysis demonstrated azoospermia with normal ejaculate volume and $\mathrm{pH}$, laboratory studies showed FSH 7.7mIU/mL (normal 1.6-8.0 mIU/mL), luteinizing hormone (LH) $5.3 \mathrm{mIU} / \mathrm{mL}$ (reference range 1.5-9.3 mIU/mL), T 616ng/dL (reference range 150-1100ng/dL). History revealed no prior fertility and no risk factors for azoospermia listed in Table 1. Physical exam revealed 12cc testicles (normal 15-20cc), palpable vasa deferentia bilaterally, flat epididymis bilaterally, and bilateral grade II varicoceles. Genetic testing showed karyotype 46XY. Assessment favored NOA secondary to bilateral varicoceles given azoospermia in the presence of low testis volume and varicocele. However, the presence of a high-normal serum FSH made the diagnosis less certain. Plan was to repair both varicoceles and perform simultaneous intraoperative testis biopsy via open excision for prognostic purposes. The bilateral varicoceles were successfully repaired, however biopsy specimen ultimately revealed normal spermatogenesis (100\% of tubules with mature sperm), favoring OA (Figure 1).

Repeat semen analysis at three months redemonstrated normal ejaculate volume, $\mathrm{pH}$, and azoospermia. Upon reassessment it was determined that the best course of action was to perform surgical reconstruction six months after varicocele repair. No sperm was visualized in the vas fluid on intraoperative microscopy and scarred, flat epididymal tubules without sperm were encountered on one side, ultimately leading to a crossed VE in which the vas deferens was 
anastomosed to the head of the contralateral epididymis. One year follow-up after crossed VE showed persistent azoospermia with plan for sperm retrieval.

\section{Discussion}

FSH and testis volume are of tremendous diagnostic value in cases of azoospermia and can correctly diagnose $96 \%$ of cases of OA without the need for testicular biopsy. ${ }^{12}$ However, we believe that our case falls within the $4 \%$ of cases of OA for which FSH and testis volume alone were insufficient to make an accurate diagnosis. Testis volume below $15 \mathrm{cc}$ in our patient favored NOA while normal range FSH favored neither OA or NOA. Our case was further complicated by the presence of bilateral varicoceles, favoring a diagnosis of NOA. Current guidelines in both the US and Canada call for diagnostic testicular biopsy for patients with normal testicular size, at least one palpable vas deferens and a normal serum follicle-stimulating hormone level. ${ }^{5,9}$ Our patient fit only one of these criteria, normal FSH, and lacked any obvious source of obstruction on history and physical exam. Varicocele is a known cause of NOA and varicocele repair has been shown to return sperm to the ejaculate or improve sperm retrieval outcomes in men with NOA and varicocele. ${ }^{8}$ Given the low suspicion of obstruction in our patient and the combined findings of low testis volume, bilateral varicoceles, and normal FSH, varicocele repair was a reasonable therapeutic step for presumed NOA. However, once obstruction was confirmed via biopsy taken at the time of varicocele repair, management of $\mathrm{OA}$ in a patient status-post bilateral varicocele repair became more challenging. In a 2013 paper, Herrel et al. detail the challenges presented by varicocele when planning for surgical repair of OA. ${ }^{13}$ The authors state that performing both procedures simultaneously could result in poor venous return and testis atrophy or loss as varicocelectomy ligates all venous return from the testis with the exception of the vasal veins. When a vasectomy is performed, preservation of some venous return must be maintained during varicocelectomy such as the cremasteric veins. Ideally, the vasectomy reversal is completed approximately 6 months or more prior to varicocelectomy. ${ }^{13}$ It should be noted that in a case series of 10 patients, vasal reconstruction and microsurgical varicocele repair were performed simultaneously. None of these 10 patients postoperative testicular atrophy, however the authors endorsed this combined procedure only for the most experienced microsurgeons. ${ }^{14}$ Ultimately the decision to perform crossed VE six months after varicocele repair in our patient was reached due to persistent azoospermia and to allow the formation of collateral venous drainage from the testes.

\section{Conclusion}

We believe that this case is important to discuss because it represents a diagnostically challenging presentation of azoospermia. We also present a management algorithm designed specifically for cases of azoospermia and varicocele with particular attention paid to cases with normal FSH (Figure 2). Our hope is that practitioners will approach these case with caution and 
err towards histological diagnosis of NOA prior to proceeding with varicocele repair in order to avoid a more complicated surgical repair of OA. 


\section{References}

1. Jarow JP, Espeland MA, Lipshultz LI. Evaluation of the azoospermic patient. $J$ Urol. 1989;142(1):62-65. http://www.ncbi.nlm.nih.gov/pubmed/2499695. Accessed August 13, 2017.

2. Jow W. Motile sperm in human testis biopsy specimens. J Androl. 1993;14(3):194-198. doi:10.1111/j.1939-4640.1993.tb00380.x.

3. Committee P, Society A. The management of infertility due to obstructive azoospermia. Fertil Steril. 2008;90(5 SUPPL.):121-124. doi:10.1016/j.fertnstert.2008.08.096.

4. Wosnitzer M, Goldstein M, Hardy MP. Review of Azoospermia. Spermatogenesis. 2014;4(March):e28218. doi:10.4161/spmg.28218.

5. Jarow J, Sigman M, Kolettis PN, et al. The Optimal Evaluation of the Infertile Male : AUA Best Practice Statement. AUA Best Pract Statement. 2011:39.

6. Schrepferman CG, Carson MR, Sparks AET, Sandlow JI. Need for sperm retrieval and crypreservation at vasectomy reversal. J Urol. 2001;166(5):1787-1789. doi:10.1016/S0022-5347(05)65676-5.

7. Bernie AM, Mata DA, Ramasamy R, Schlegel PN. Comparison of microdissection testicular sperm extraction, conventional testicular sperm extraction, and testicular sperm aspiration for nonobstructive azoospermia: a systematic review and meta-analysis. doi:10.1016/j.fertnstert.2015.07.1136.

8. Weedin JW, Khera M, Lipshultz LI. Varicocele Repair in Patients With Nonobstructive Azoospermia: A Meta-Analysis. J Urol. 2010;183(6):2309-2315. doi:10.1016/j.juro.2010.02.012.

9. Jarvi K, Lo K, Grober E, et al. CUA Guideline: The workup and management of azoospermic males cua guideline. Can Urol Assoc J. 2015;99(August):229-235. doi:10.5489/cuaj.3209.

10. Gudeloglu A, Parekattil SJ. Update in the evaluation of the azoospermic male. Clinics (Sao Paulo). 2013;68 Suppl 1(Suppl 1):27-34. doi:10.6061/clinics/2013(sup01)04.

11. Chiba K, Enatsu N, Fujisawa M. Management of non-obstructive azoospermia; Management of non-obstructive azoospermia. doi:10.1007/s12522-016-0234-z.

12. Schoor RA, Elhanbly S, Niederberger CS, Ross LS. The role of testicular biopsy in the modern management of male infertility. J Urol. 2002;167(1):197-200. http://www.ncbi.nlm.nih.gov/pubmed/11743304. Accessed February 14, 2018.

13. Herrel L, Hsiao W. Microsurgical vasovasostomy. Asian J Androl. 2013;15(1):44-48. doi:10.1038/aja.2012.79.

14. Mulhall JP, Stokes S, Andrawis R, Buch JP. Simultaneous microsurgical vasal reconstruction and varicocele ligation: Safety profile and outcomes. Urology. 1997;50(3):438-442. doi:10.1016/S0090-4295(97)00290-2. 


\section{Figures and Tables}

Fig. 1. Normal spermatogenesis found on intraoperative testis biopsy during bilateral varicocele repair.



Fig. 2. Non-obstructive etiology of etiology should be confirmed via diagnostic biopsy in patients with azoospermia, varicocele, and normal FSH and testis volume prior to varicocele repair. Varicocele repair should not be performed in patients with histological evidence of OA found on diagnostic biopsy.

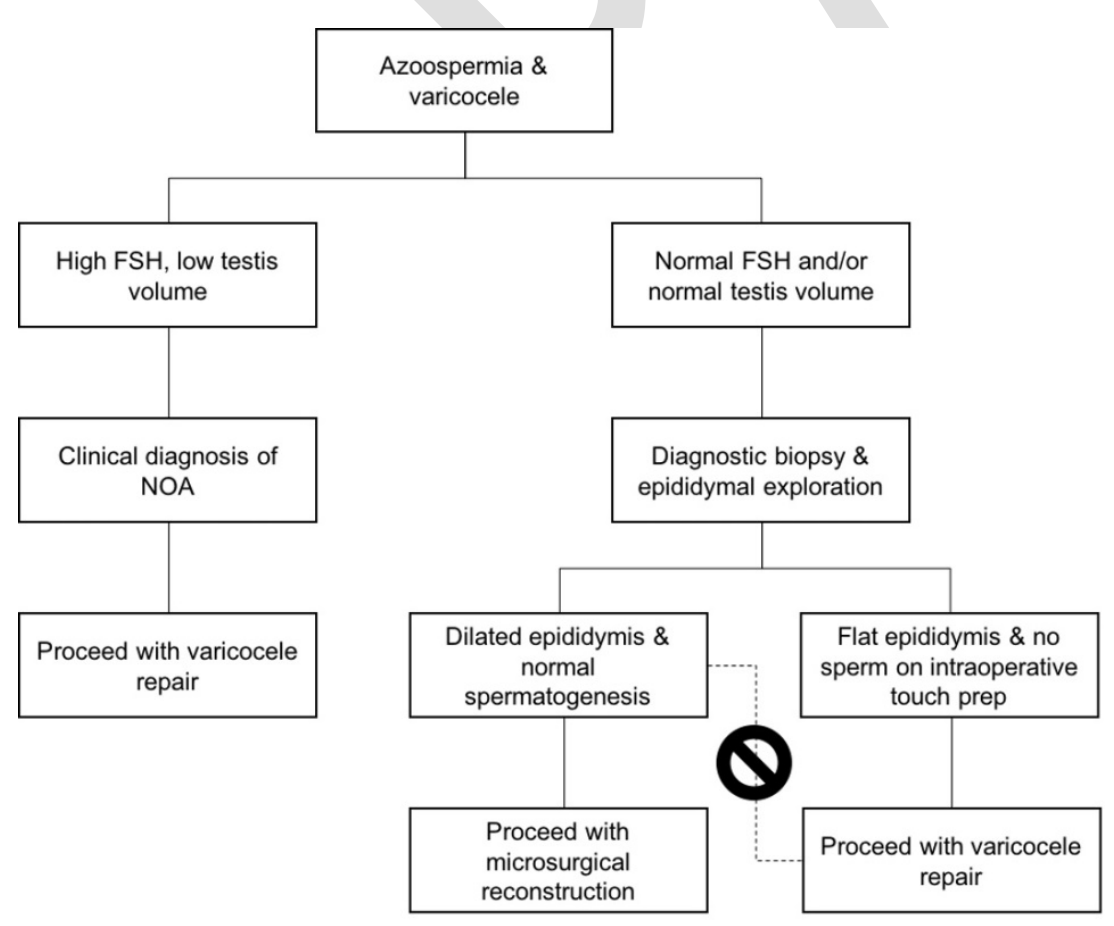




\begin{tabular}{|l|}
\hline Table 1. Relevant medical history in the evaluation of azoospermia \\
\hline Prior fertility \\
Childhood illnesses, such as viral orchitis or cryptorchidism \\
\hline Genital trauma or prior pelvic or inguinal surgery \\
\hline Infections, such as epididymitis or urethritis \\
\hline Gonadotoxin exposures, such as prior radiation therapy/chemotherapy \\
\hline Recent fever or heat exposure \\
\hline Current medications \\
\hline Family history of birth defects \\
\hline Mental retardation \\
\hline Reproductive failure \\
\hline Cystic fibrosis
\end{tabular}

